\title{
Influence of Diet, Sex, and Viral Infections on the Gut Microbiota Composition of Spodoptera exigua Caterpillars
}

OPEN ACCESS

Edited by:

George Tsiamis,

University of Patras, Greece

Reviewed by:

Antonios Alekos Augustinos, Hellenic Agricultural Organization,

Greece

Panagiota Stathopoulou

University of Patras, Greece Rosa Maria Murillo,

Public University of Navarre, Spain

*Correspondence:

Salvador Herrero

salvador.herrero@uv.es

Specialty section:

This article was submitted to Systems Microbiology,

a section of the journal

Frontiers in Microbiology

Received: 16 January 2020

Accepted: 30 March 2020

Published: 06 May 2020

Citation:

Martínez-Solís M, Collado MC and Herrero S (2020) Influence of Diet, Sex, and Viral Infections on the Gut Microbiota Composition

of Spodoptera exigua Caterpillars.

Front. Microbiol. 11:753

doi: 10.3389/fmicb.2020.00753

\begin{abstract}
María Martínez-Solís ${ }^{1}$, María Carmen Collado ${ }^{2}$ and Salvador Herrero ${ }^{1 *}$
${ }^{1}$ Estructura de Recerca Interdisciplinar en Biotecnologia i Biomedicina (ERI BIOTECMED), Departamento de Genética, Universitat de València, Valencia, Spain, ${ }^{2}$ Instituto de Agroquímica y Tecnología de Alimentos, Consejo Superior de Investigaciones Cientificas (IATA-CSIC), Valencia, Spain
\end{abstract}

The gut microbiota plays essential roles in processes related with metabolism, physiology, and immunity in all organisms, including insects. In the present work, we performed a broad analysis of the Spodoptera exigua gut microbiota, a major agricultural pest. We analyzed the influence of multiple parameters such as diet, geographic location, sex, or viral infections on S. exigua caterpillar gut microbiota composition. Our study revealed a high variability in bacterial composition among individuals, and a major influence of environmental bacteria (including those acquired through diet) on the gut microbiota composition, supporting previous studies that claim resident microbiota are lacking in caterpillars. Previous studies with laboratory-reared insects showed that changes in caterpillar gut bacterial composition affect the insecticidal properties of entomopathogenic viruses and bacteria. Our study revealed different microbiota composition in field insects carrying a natural viral infection with Spodoptera exigua nucleopolyhedrovirus (SeMNPV) and/or Spodoptera exigua iflavirus 1 (SelV1). Few taxa can be specifically associated with the infection, suggesting microbiota influence the infective process of these natural pathogens, and providing new strategies for insect pest management.

Keywords: lepidoptera, microbiota, Spodoptera exigua, 16S rRNA, viral infection

\section{INTRODUCTION}

The Lepidoptera Spodoptera exigua (Hübner) (Noctuidae) is an important polyphagous agricultural pest, polyphagous and worldwide distributed. Traditionally, chemical insecticides have been used to control this pest but, extensive use over a long time period has led to the emergence of resistant populations (Brewer and Trumble, 1989; Moulton et al., 2000; Osorio et al., 2008; Ahmad and Arif, 2010; Che et al., 2013). Therefore, biological insecticides such as baculovirus and Bacillus thuringiensis (Bt) represent an effective and safer alternative for pest control.

Gut microbiota is described as the complex community of microorganisms living in the digestive tracts of human and other animals in a symbiotic relationship. Many animals, including insects, are colonized by microbial symbionts, which can regulate host processes related with development, immunity, and metabolism (Dillon and Dillon, 2004; Engel and Moran, 2013). Most caterpillars are herbivorous, and their gut bacteria can play important roles in nutrition and host adaptation. 
For instance, gut microbiota in caterpillars can contribute to plant cell wall digestion (Mason et al., 2015; Xia et al., 2017), detoxification of toxic compounds synthesized by the plants they feed on (Shao et al., 2011; Hammer and Bowers, 2015), or even suppress plant defense mechanisms (Acevedo et al., 2017). In recent years, researchers investigated if caterpillars depend on their gut microbes for feeding and/or development, but this question remains unclear. While some studies have shown that diet affects the insect microbiome composition (Berman et al., 2018; Jones et al., 2019), others have reported that diet does not have a clear effect on gut microbiota composition (Whitaker et al., 2016; Hammer et al., 2017). Moreover, the great variability usually observed in lepidopteran bacterial composition also indicates that many factors influence the final gut bacterial composition of these insects (reviewed in Mereghetti et al., 2017).

Furthermore, the Lepidoptera gut microbiota seems to influence the host interaction with pathogenic microorganisms. Several studies on different lepidopteran species revealed changes in gut microbiota composition after intoxication with $\mathrm{Bt}$ toxins (Broderick et al., 2004; Caccia et al., 2016), and insect susceptibility alteration to Bt and its toxins (Broderick et al., 2004) due to gut microbiota changes. In addition, Xia et al. (2013) suggested that certain bacterial taxa can confer Bt-resistance in Plutella xylostella larvae. In the case of S. exigua, our previous studies showed an increase in Bt tolerance associated with an increase in the gut bacterial load (Hernández-Martínez et al., 2010). Regarding viral pathogens such as baculovirus, a previous study from our laboratory revealed an increase in gut bacterial load after infection of a laboratory-reared $S$. exigua colony with its baculovirus, Spodoptera exigua multiple nucleopolyhedrovirus (SeMNPV) (Jakubowska et al., 2013). Such change in bacterial load was associated with an increase in baculovirus virulence, pathogenicity, and dispersion.

Baculoviruses are DNA viruses highly specific against different invertebrate species which cause larval death after viral ingestion. In addition to the oral infection caused by baculovirus, the virus can asymptomatically persist on the insects and be vertically transmitted to the offspring (Virto et al., 2014). In the case of S. exigua, 54\% of field adult insects are covertly infected with its baculovirus, SeMNPV (Virto et al., 2014). In addition, some field insects can also carry a second covert infection with RNA viruses from the Iflaviridae family, which apparently do not cause lethal infection. Spodoptera exigua Iflavirus 1 (SeIV1) (MillánLeiva et al., 2012) was present in about $13.1 \%$ of field insects and $6.1 \%$ of insects were simultaneously infected with SeMNPV and SeIV1 (Virto et al., 2014). These covertly infectious viruses can be activated by different stress factors, leading to a lethal infection and finally killing the insect.

Based on the previous observations mentioned above, we hypothesized the possible role of gut microbiota composition in modulating $S$. exigua interaction with its naturally occurring viruses in the field. In the present work, as a first step in this investigation, we characterized the gut microbiota composition of laboratory and field collected $S$. exigua caterpillar, and studied the influence of dietary regimen, geographic location, and caterpillar sex on the gut microbiota composition. In a second part of the study, we analyzed viral infection interaction and gut microbiota composition, identifying certain bacterial groups which could shape viral-host interaction.

\section{MATERIALS AND METHODS}

\section{Insects}

In this study, samples came from $S$. exigua larvae with different backgrounds and dietary regimens. Field larvae were obtained from pepper greenhouses located in the Almería province (Spain). Third to fifth instar larvae were collected during September and October 2015 and sent to our laboratory in Valencia (Spain). The insects were sent in individual plastic bottles containing the pepper leaf from where they were collected, and the larvae were dissected and processed immediately upon arrival. The insects reared on standard artificial diet (AD) (Elvira et al., 2010) came from our laboratory colony, which has been maintained for more than 200 generations. The colony is maintained at $25 \pm 3^{\circ} \mathrm{C}$ with $70 \pm 5 \%$ relative humidity and a $16 / 8 \mathrm{~h}$ (light/dark) photoperiod. We also used S. exigua larvae from our laboratory colony fed a plant-based diet (PBD), which was prepared with lyophilized plant leaves in $2 \%$ agar and supplemented with 5\% AD. For the PBD studies, fourth instar larvae previously reared on $\mathrm{AD}$ were transferred to the $\mathrm{PBD}$ and reared for $48 \mathrm{~h}$ before dissection. In parallel, a group of larvae were kept only on $\mathrm{AD}$ and dissected simultaneously. The plants employed for the PBD studies were pepper (dulce de España variety) and two different tomato varieties [Ailsa (A) and Money Maker (MM)]. Plants were grown in greenhouse facilities and harvested before floriation.

The S. exigua larvae were dissected and processed as follows. The whole gut (including the gut content) of each individual larva from the field, and pools of three guts per sample from an AD and PBD were dissected and homogenized in Luria-Bertani (LB) medium supplemented with $10 \%$ glycerol and frozen at $-80^{\circ} \mathrm{C}$ for DNA extraction. For each sample, half of the remaining body was directly frozen at $-80^{\circ} \mathrm{C}$ for subsequent DNA extraction, and the other half was frozen in RNAzol ${ }^{\circledR}$ RT reagent (Sigma Aldrich, St Louis, MO, United States) at $-80^{\circ} \mathrm{C}$ for RNA extraction.

\section{DNA Extraction and Sequencing}

A fraction of each homogenized gut was used for total DNA extraction using the MasterPure ${ }^{\mathrm{TM}}$ Complete DNA \& RNA Purification Kit (Epicenter, Madison, WI, United States) according to the manufacturer's instructions. Purified DNA was quantified in QUBIT and $10 \mathrm{ng} / \mathrm{ul}$ were used for amplification and sequencing of the $16 \mathrm{~S}$ rRNA gene from 77 samples. The amplicon sequencing protocol targets the $16 \mathrm{~S}$ gene V3 and V4 regions (459 bp), with the primers designed surrounding conserved regions (Klindworth et al., 2013). Following the Illumina amplicon libraries protocol, DNA amplicon libraries were generated using a limited cycle PCR: initial denaturation at $95^{\circ} \mathrm{C}$ for $3 \mathrm{~min}$, followed by 25 cycles of annealing $\left(95^{\circ} \mathrm{C}\right.$ $30 \mathrm{~s}, 55^{\circ} \mathrm{C} 30 \mathrm{~s}, 72^{\circ} \mathrm{C} 30 \mathrm{~s}$ ), and extension at $72^{\circ} \mathrm{C}$ for $5 \mathrm{~min}$, using a KAPA HiFi HotStart ReadyMix (KK2602). Then Illumina sequencing adaptors and dual-index barcodes (Nextera XT index kit v2, FC-131-2001) were added to the amplicon. Libraries 
were then normalized and pooled prior to sequencing. The pool containing indexed amplicons was then loaded onto the MiSeq reagent cartridge v3 (MS-102-3003) spiked with 10\% PhiX control to improve base calling during sequencing, as recommended by Illumina for amplicon sequencing. Sequencing was conducted using a $2 \times 300$-pb paired-end run on an Illumina MiSeq sequencing system.

\section{Sex Determination}

Field larvae sex was molecularly determined by kettin gene copy number relative quantification. Kettin is a sex-linked gene without dosage compensation as previously described for Bombyx mori (Suzuki et al., 1999), and was recently used to determine sex in different Lepidopteran larvae species (Belousova et al., 2019). In this study, specific primers for S. exigua kettin gene amplification were designed (Supplementary Table 1) using the same region published previously for B. mori (Koike et al., 2003). Primers were previously validated on thorax DNA obtained from S. exigua male and female adults.

Total DNA was extracted from field larvae carcasses using the MasterPure ${ }^{\mathrm{TM}}$ Complete DNA \& RNA Purification Kit (Epicenter, Madison, WI, United States), and the isolated DNAs (50 ng) were used for kettin quantification by quantitative PCR (qPCR) using the specific primers and ATP synthase as a reference. The qPCR was performed with 5x HOT FIREPOL EvaGreen qPCR Mix Plus (ROX) (Solis BioDyne, Tartu, Estonia) following standard protocols, and the DNA amplification was measured in a StepOnePlus Real-Time PCR System (Applied Biosystems, Foster City, CA, United States). The relative quantification was represented as $2^{-\Delta \Delta C t}$ calculated according to the method described in Livak and Schmittgen (2001) and Belousova et al. (2019), were $\Delta \Delta \mathrm{Ct}=\left(\mathrm{Ct}_{\text {kettin }}-\mathrm{Ct}_{\text {ATP synthase }}\right)$ $\operatorname{Avg}\left(\mathrm{Ct}_{\text {kettin }}-\mathrm{Ct}_{\text {ATP synthase }}\right)_{\text {female }}$.

\section{RNA Extraction and Virus Quantification}

The presence of the SeIV1 RNA virus and the active replication of the SeMNPV DNA virus in field insects were determined by qPCR using specific primers (Supplementary Table 1). Total RNA was isolated from the carcass of each larvae using RNAzol ${ }^{\circledR}$ RT reagent (Sigma Aldrich, St Louis, MO, United States) following the manufacturer's protocol, and used for cDNA synthesis using the PrimeScript RT Reagent kit (TaKaRa Bio Inc., Otsu Shiga, Japan). The qPCR was performed as described in the previous section. The amplification curve of each sample was examined, and $\Delta \mathrm{Rn} \geq 1$ values were considered as positive viral infections.

\section{Total Bacterial Load Quantification}

The total bacterial load was quantified in the field samples by qPCR using $16 \mathrm{~S}$ rRNA universal primers (Nadkarni et al., 2002). The qPCR was performed with total DNA (50 ng) isolated from the gut of each larvae in a StepOnePlus realtime PCR system (Applied Biosystems, Foster City, CA, United States). The bacterial concentration was calculated by comparison with a standard curve of known bacterial DNA quantities, and then statistically analyzed for viral presence with a Student's $t$-test (GraphPad Prism version 7.00) as described in Gasmi et al. (2019).

\section{Data Analysis}

Data-mining and statistical analysis were performed with the open-source software QIIME (v. 1.9) (Caporaso et al., 2010) and the online Calypso pipeline (v. 8.84) (Zakrzewski et al., 2017). Quality assessment of obtained reads was carried out with the prinseq-lite program (Schmieder et al., 2011) with defined parameters (i.e., min_length, 50; trim_qual_right, 20; trim_qual_type, mean; trim_qual_window, 20). Paired reads from Illumina sequencing were joined using fastq-join from the ea-tools suite (Aronesty, 2011). Potential chimeric sequences were removed using USEARCH 6.0 available at RDPipeline (Edgar et al., 2011). Filtered and demultiplexed sequences were then processed with QIIME software using default parameters. First, the sequences were clustered into operational taxonomic units (OTUs) using de novo OTU picking (pick_de_novo_otus.py script) based on $97 \%$ identity and filtering the unassigned, mitochondria, chloroflexi, and cyanobacteria taxa using the QIIME's filter_taxa_from_otu_table.py script, to visualize the most abundant phyla in all samples. Next, to analyze the samples more deeply to look for microbiotia differences, the generated OTU table was filtered to include only the four most abundant phyla. Bacterial composition was analyzed through the summarize_taxa_through_plots.py script and it was represented as the relative abundance of the 20 most abundant genera in a bar graphic using Excel software. We also identified the core microbiome (compute_core_microbiome.py) as those OTUs present in at least $50 \%$ of all samples.

The OTU table including only the most abundant phyla was filtered in Calypso software to remove the samples with less than 1000 reads from the analysis, and the resulting data was transformed by CSS (cumulative-sum scaling)+log with total sum normalization (TSS). Then, canonical correspondence analysis (CCA) was used to estimate differences in microbiota (at the genus level) according to different factors (diet, sex, or viral infections). Alpha diversity and richness were determined at the genus level using the Shannon index and Chaol index, respectively. The Wilcoxon rank test was employed to identify differentially abundant bacteria associated with viral presence. Spearman correlation analysis using GraphPad Prism (version 7.00) were used to examine the relationship between the total bacterial loads and the diversity or richness in field samples.

\section{RESULTS}

\section{S. exigua Caterpillar Microbiota Compositions}

A total of 3,450,084 reads were obtained from $16 \mathrm{~S}$ rRNA gene Illumina Miseq sequencing of $77 \mathrm{~S}$. exigua samples (45 samples from field captures, 15 from $\mathrm{AD}$, and 17 from the PBD) (detailed on Supplementary Tables 2,3). After cleaning and chimera filtration, the remaining 2,795,707 reads led to the identification of 42,601 OTUs. After removing some host $S$. exigua contaminant sequences (designated as 
unassigned), mitochondria, chloroflexi, and cyanobacteria taxa, 21,197 OTUs remained. These OTUs were assigned mostly to Proteobacteria, Firmicutes, Bacteroidetes, and Actinobacteria phyla (98\% total phyla coverage) showing different distribution across the analyzed groups (Figure 1). Proteobacteria was the most abundant phyla in all groups, representing around 50\% or more OTUs in the different groups. The phylum Firmicutes was almost as abundant as Proteobacteria in the $\mathrm{AD}$ group but decreased to around $20 \%$ in the larvae fed PBD group. Bacteroidetes was the least abundant in all groups, although its presence was slightly higher in field samples than from laboratory larvae. Actinobacteria was more abundant in samples from two tomato varieties. Additionally, 8 samples (5 field samples, $1 \mathrm{AD}$, 1 from tomato (MM), and 1 from pepper) were removed from the analysis, since they contained less than 1000 reads. Although some variability in the richness and Shannon indices between the individual samples was observed (Supplementary Figures 1A,B), rarefaction curves tended to reach a plateau indicating that the sequencing depth was enough to capture the majority of microbial diversity (Supplementary Figure 1C).

\section{Changes in Gut Microbiota Associated With Different Diets}

Influence of diet on the bacterial community composition was further characterized according to their genus distribution (Figure 2) by analyzing bacterial composition changes in caterpillar reared on $\mathrm{AD}$, and then transferred to a $\mathrm{PBD}$ for 48 h. A multivariate canonical correspondence analysis (CCA) at the genus level revealed significant differences $(P=0.002)$ among the different diet types. Accordingly, changes in diet composition produced a quick shift in gut microbial composition, suggesting that $S$. exigua larvae microbiota composition was strongly influenced by ingested food. Composition differences were observed even among larvae fed (for $48 \mathrm{~h}$ ) with two different tomato varieties (Figure 2A).

The bacterial diversity measured by the Shannon index at the genus level was almost identical for larvae fed a PBD, showing very little variability among samples (Figure 2B). In contrast, those larvae fed an $\mathrm{AD}$ showed an apparent greater variability among each sample. Nevertheless, no significant differences in bacterial diversity were observed between the different diets tested. The Chaol index for richness estimation also did not show differences between diets (Figure 2C). Further bacterial composition characterization was carried out by relative abundance comparison of the 20 most abundant genera for each sample (Figure 2D). Great genus composition heterogeneity was observed and, consequently, no clear association of specific genera to the different diets could be established.

\section{Gut Microbiota Composition in Field S. exigua Larvae}

S. exigua bacterial composition was also evaluated in individual larvae collected from pepper plants from different greenhouses in the Almería province (Spain). Relative abundance analysis of the 20 most abundant genera revealed great similarity among individuals collected from the same greenhouse (Figure 3).
TABLE 1 | The most common OTUs from the gut microbiota of S. exigua larvae.

\begin{tabular}{lc}
\hline OTU & Prevalence \\
\hline P_Bacteroidetes_f_Enterobacteriaceae_1157
\end{tabular}

However, samples from different greenhouses were highly diverse in composition. These results suggest a major influence of environment (including the ingested plant) on S. exigua larvae gut bacterial composition.

\section{Core Microbiome}

A core microbiome was defined as those OTUs present in at least $50 \%$ of all samples. Taking all samples individually into account, a core that comprises 20 OTUs belonging to phyla Proteobacteria was identified (Table 1). However, no bacteria (OTU) were found in common for all samples analyzed. In addition, a core microbiome was also analyzed in a more restricted sample group, the field-collected insects. In that case, only one OTU, belonging to the Pseudomonas genus, was found in common in all the field individuals. Results obtained with the two sample sets analyzed revealed the lack of a true core microbiome in the S. exigua caterpillar, since no OTUs were found in common between all the analyzed samples.

\section{Sex Influence on Gut Microbiota Composition}

Although caterpillars do not show obvious sex dimorphism, the immature reproductive organs are already present at the larval stage. We wondered if caterpillar sexual destination could influence microbiota composition. To test such hypothesis, the gut microbiota composition from field collected S. exigua larvae were also analyzed according to their sex. Sex determination was performed by kettin gene quantification in field samples, and 12 males and 23 females were identified (5 samples could not be sexed due lack of proper DNA) (Figure 4A). The bacterial community was examined using a CCA at the genus level to assess 

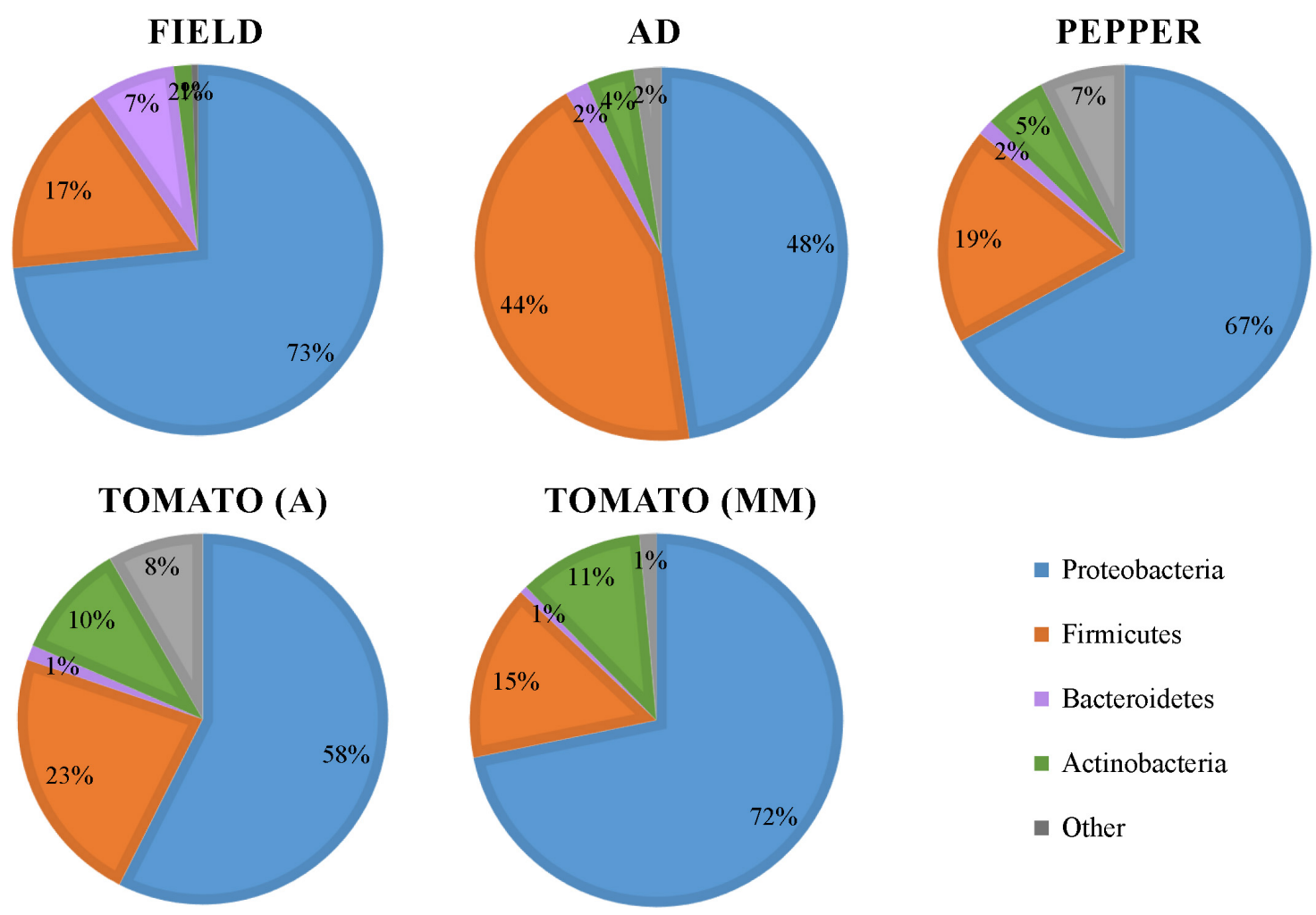

FIGURE 1 | Bacterial phyla distribution in the gut of $S$. exigua. Pie charts represent the relative abundance in percentages of the main phyla found in the gut of S. exigua larvae collected on the field from pepper greenhouses and the laboratory colony fed with different diets (AD: artificial diet; A: Ailsa variety; MM: Money Maker variety).

if larvae microbiota differs depending on sex, but no significant differences were found (Figure 4B). Additionally, the diversity and richness indices also did not show differences between sexes (Figures 4C,D).

\section{Gut Bacterial Composition and Viral Infections}

Naturally present viruses in the S. exigua field samples were determined by qPCR amplification of specific viral genes. We found an infectious rate around 50\%, which was distributed as follows: 6 larvae infected with BV, 9 larvae infected with SeIV1, and 6 larvae simultaneously infected by both viruses, whereas 19 larvae were classified as virus-free. The gut microbiota was then examined at the genus level according to the presence or absence of viral infection. Although the Shannon and the Chaol indices estimated that diversity and richness, respectively, were roughly the same for virus-infected and non-virus-infected samples (Figures 5B,C), the CCA analysis showed a significant difference $(P=0.003)$ in microbiota composition (Figure 5A).

An additional analysis revealed the existence of 9 OTUs significantly associated $(P<0.05)$ and more abundant (at least twice) on the viral infected samples (Table 2 and Supplementary Figure 2), the sequences of which are found in the supplementary data. Six of the identified OTUs belong to phylum Proteobacteria (three from the genus Acinetobacter), two OTUs were identified as bacteria from genus Pedobacter (phylum Bacteroidetes), and another from phylum Firmicutes. An interesting observation is that all OTUs which showed significant abundance changes were overrepresented in the virusinfected group. A moderate increase in abundance of less than 10 -fold was found for all the identified OTUs, except for the p__Proteobacteria_g_Acinetobacter_12048 which is 36,120fold more abundant in the virus-infected group. Nevertheless, no differences in bacterial load were observed between the viralinfected and non-infected caterpillars (Figure 5D). Interestingly, a deeper analysis revealed a significant negative correlation between the bacterial load and the diversity index for both groups (Figure 5E). However, the relationship between bacterial load and richness only showed a significant positive correlation for the viral infected samples (Figure 5F), which suggests that an increase in microbiota in viral-infected samples leads to the presence of a greater number of bacterial taxa.

\section{DISCUSSION}

In this work, we performed a comprehensive characterization of $S$. exigua larvae gut bacterial microbiota composition, and determined how aspects such as diet, location, sex, and viral presence influence it. Taxonomic analysis revealed that 
A

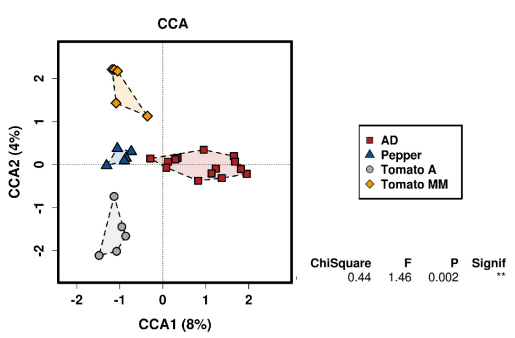

B

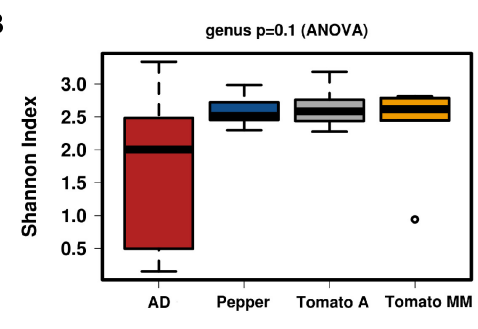

C

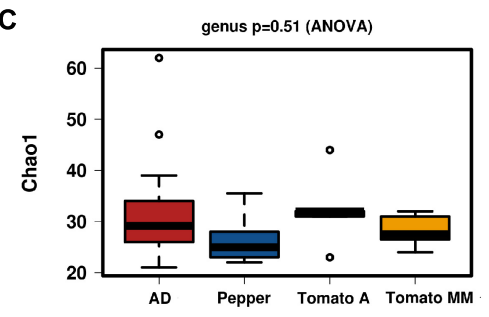

D

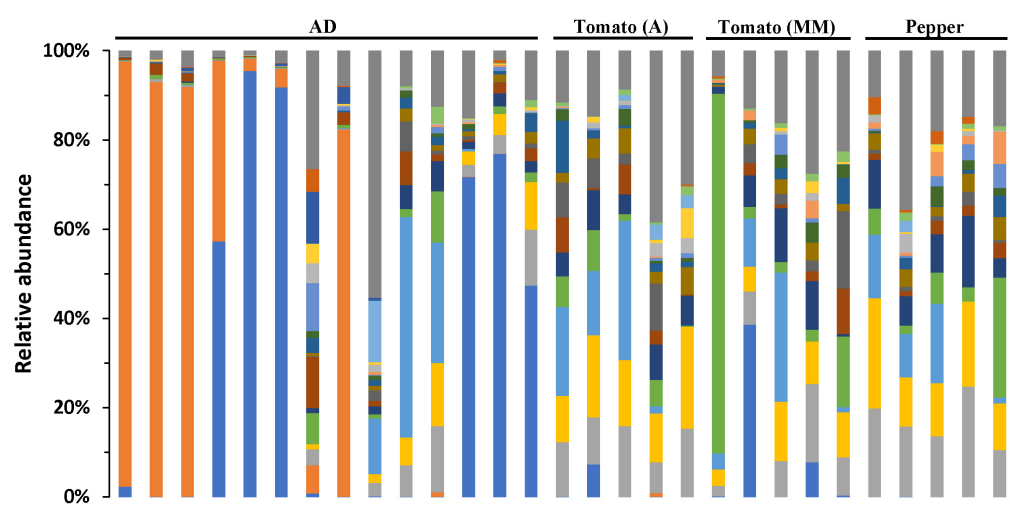

- Others

Lysinibacillus

- Unclassified Phyllobacteriaceae

- Coprothermobacter

Pseudomonas

Unclassified Comamonadacea

- Veillonella

Sphingomona

- Acinetobacter

Rhizobium

Rubrobacte

- Bacillus

- Ralstonia
-Unclassified Enterobacteriaceae 1

- Staphylococcus

Unclassified Oxalobacteraceae

Delftia

- Enterococcus

- Unclassified Enterobacteriaceae 2

FIGURE 2 | Microbiota of S. exigua larvae according the diet. Canonical correspondence analysis (CCA) (A), estimation of the Shannon index diversity (B), the Chao1 index of richness (C) and the relative abundance of the 20 most abundant genera in S. exigua larvae from the laboratory colony fed with different diets (D) at a genus level.

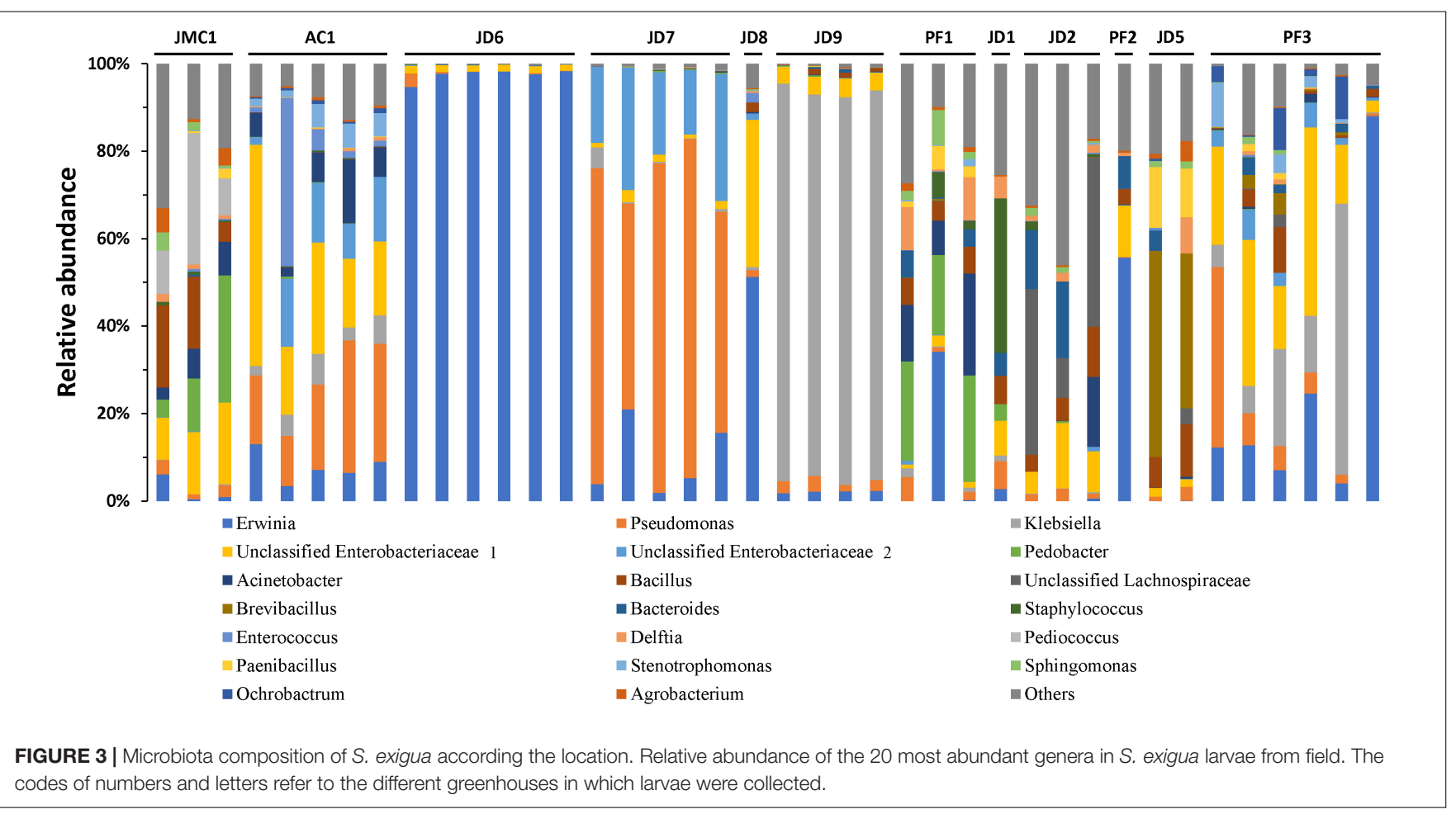

the $S$. exigua gut bacterial community independent of the feeding conditions (diet and origin), was mainly composed of Proteobacteria, Firmicutes, Bacteroidetes, and Actinobacteria, as previously described by Gao et al. (2019). These four phyla were also the most common found in other lepidopteran species (Whitaker et al., 2016; Xia et al., 2017; Chen et al., 2018; 
A

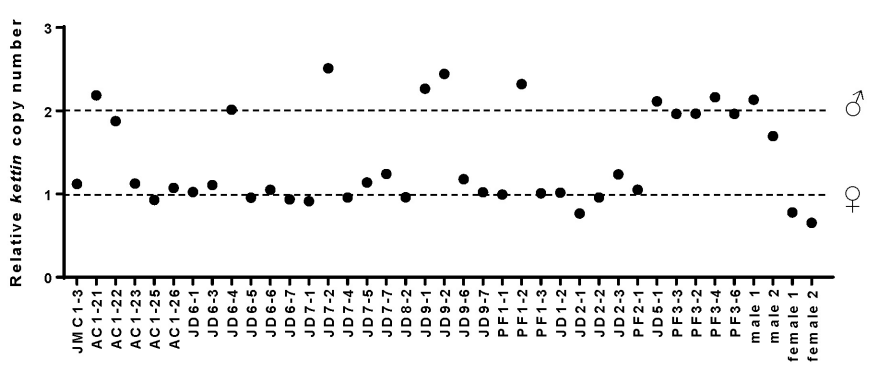

C

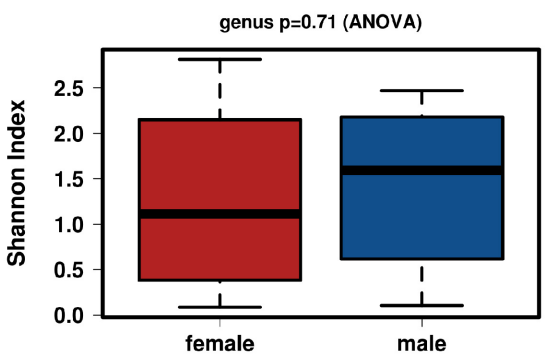

B

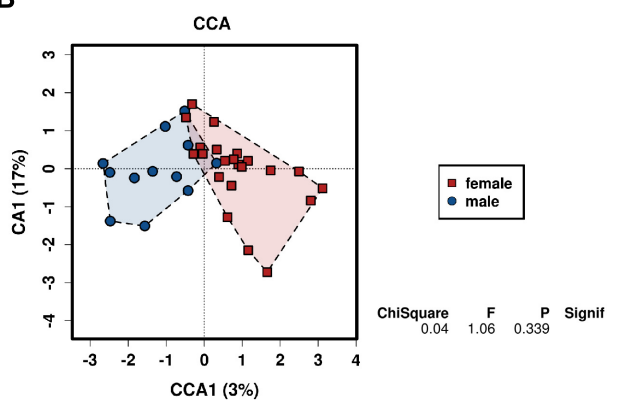

D

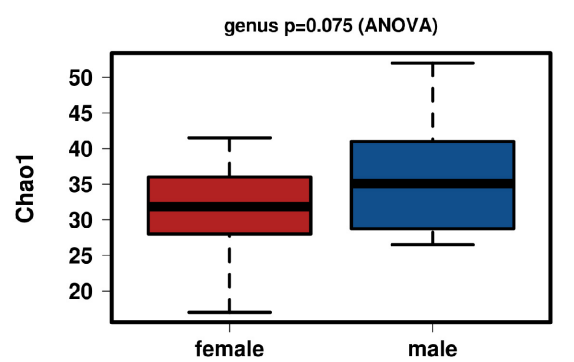

FIGURE 4 | Influence of sex on the microbiota of S. exigua. Sex determination of the S. exigua larvae from field by quantification of the kettin gene copy number (A). Canonical correspondence analysis (B), the estimation of the Shannon index diversity (C) and the Chao1 index for richness (D) at a genus level according the sex of the collected larvae.

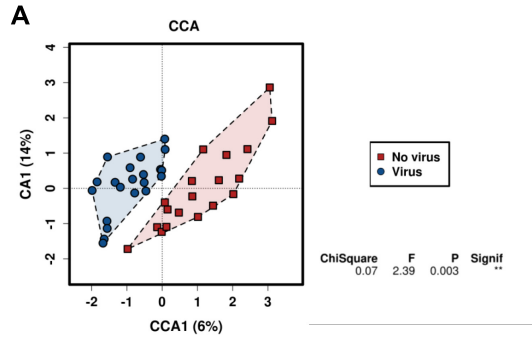

D

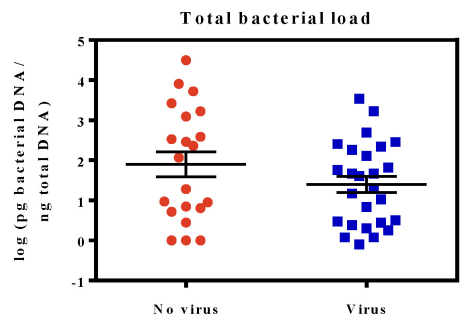

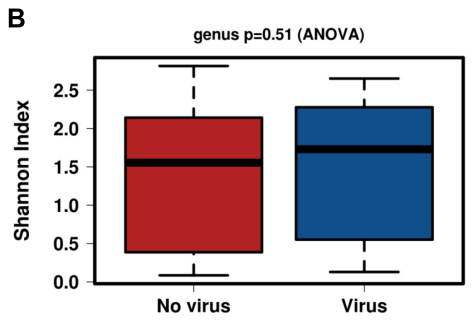

C

E

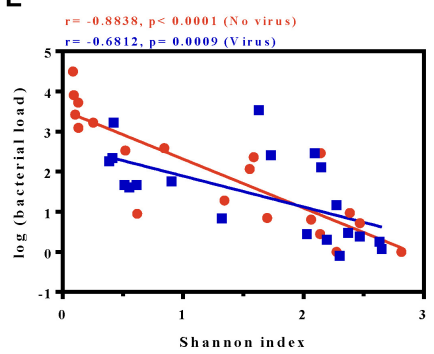

$\mathbf{F}$
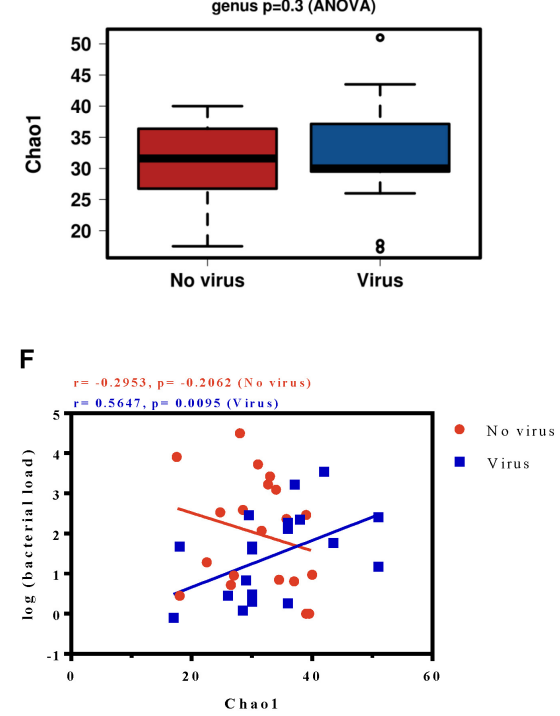

FIGURE 5 | Microbiota analysis of S. exigua larvae according their viral infection status. Canonical correspondence analysis (A), the estimation of the Shannon index diversity (B) and the Chao1 richness index (C) at a genus level in field S. exigua larvae naturally infected and non-infected with viruses. Calculation of the total bacterial load of $S$. exigua larvae from viral-infected and viral-free field samples (D) and their relationship with diversity $(\mathbf{E})$ and richness $(\mathbf{F})$. The best-fit lines, the Spearman $r$ correlations and the $p$-values of the regression are shown for each analysis.

Phalnikar et al., 2018; van Schooten et al., 2018), as well as in other insects (Yun et al., 2014). Slight abundance differences were observed between the different groups tested, but likely the main difference was the increased presence of the phylum Firmicutes in larvae reared on $\mathrm{AD}$ in comparison with those reared on a $\mathrm{PBD}$ (Figure 1). This observation suggests that diet could influence S. exigua larvae microbial gut composition. Thus, the bacterial composition was further analyzed. 
TABLE 2 | Differentially abundant OTUs associated to viral infection.

\begin{tabular}{lrc}
\hline Otu & $\begin{array}{r}\text { Fold } \\
\text { change }\end{array}$ & $\begin{array}{c}\boldsymbol{P} \text { - } \\
\text { valor }\end{array}$ \\
\hline P_Bacteroidetes_g_Pedobacter_3455 & 2.1 & 0.0020 \\
P_Bacteroidetes_g_Pedobacter_1816 & 2.8 & 0.0038 \\
P_Proteobacteria_g_Acinetobacter_s_johnsonii_15272 & 9.5 & 0.0065 \\
P_Proteobacteria_g_Acinetobacter_12048 & 36,120 & 0.0270 \\
P_Proteobacteria_g_Acinetobacter_15052 & 3.1 & 0.0330 \\
P_Firmicutes_g_Staphylococcus_1362 & 9.9 & 0.0250 \\
P_Proteobacteria_g__Sphingobium_40602 & 2.8 & 0.0250 \\
P_Proteobacteria_f_Xanthomonadaceae_11563 & 2.8 & 0.0092 \\
P_Proteobacteria_f_Enterobacteriaceae_14126 & 3.2 & 0.0370 \\
\hline
\end{tabular}

In a more detailed analysis with respect to gut bacterial composition we focused on laboratory colony larvae fed different diet types ( $\mathrm{AD}$, tomato ( $\mathrm{A}$ and $\mathrm{MM}$ varieties), and pepper). A rapid shift in gut bacterial composition was associated with diet changes. Although the relative abundance analyses of the most abundant genera showed great heterogeneity and variability, even among samples from the same diet group, a significant difference was observed in the multivariate analysis (CCA) indicating that larval gut microbiota composition is strongly influenced by diet. No significant differences were observed neither in diversity nor in richness at the genus level, however, the samples reared on $\mathrm{AD}$ showed great variability, while samples reared on a PBD were more homogeneous (Figure 2). This observation could be because those insects came from different generations collected at different periods. In addition, although the $\mathrm{AD}$ recipe was not changed, the ingredients with it is made of can be slightly differ from one batch to another and affect someway the larvae gut microbiota. Other differences between larvae reared on $\mathrm{AD}$ or PBD can be extracted from this study. For example, the genus Enterococcus and others from the Enterobacteriaceae family were more abundant in samples from $\mathrm{AD}$, as previously shown for S. exigua and other lepidopteran species (Broderick et al., 2004; Xiang et al., 2006; Raymond et al., 2009; Hernández-Martínez et al., 2010; Jakubowska et al., 2013). However, in these previous studies Enterococcus was reported as a bacterium present in all samples as the most abundant, while in our analysis Enterococcus genus abundance was highly variable among samples. Although a fast shift in microbiota composition had been observed after only $48 \mathrm{~h}$ of shifting diet, we cannot discard that longer time (e.g., at least one complete generation) could show stronger effects. In addition, previous Lepidoptera studies revealed apparent contradictions with respect to diet influence on gut microbiota. Some studies claimed diet significantly influenced lepidopteran gut microbiota composition (Yun et al., 2014; Berman et al., 2018; Phalnikar et al., 2018; Jones et al., 2019), whereas other authors reported diet had little or no effect on microbiota (Whitaker et al., 2016; Chaturvedi et al., 2017; Minard et al., 2019). This apparent contradiction seems to reveal the lack of caterpillar specific gut microbiota as reported by Hammer et al. (2017). Consequently, any diet change (and the microbiota present in the diet) or in the environment would produce a shift in gut microbiota composition without being diet specific.
In agreement with that, field larvae samples also showed great variability in gut bacterial composition, even though the caterpillars were all collected from pepper plants. Nevertheless, some general microbial composition patterns were observed when samples were grouped according to their location (Figure 3), suggesting a stronger contribution from environmental bacteria in the final larval gut composition than bacteria associated with PBD. This is consistent with a recent study by Hannula and colleagues (Hannula et al., 2019), in which they demonstrated that Mamestra brassicae larvae microbiota depends on the soil microbiome. So, the variability observed among the different greenhouses could be attributed to differences in foliar microbiota of each pepper plant and the greenhouse soil microbiota in which the caterpillars were collected. This explanation is also supported by previous studies in which the larval gut bacteria did not totally correspond with dietary associated leaf bacteria (Whitaker et al., 2016; Hammer et al., 2017; Minard et al., 2019). Thus, the authors suggested larval gut microbiota is composed of transient bacteria instead of resident microbiota, due to the intrinsic insect gut physiology, such as their high $\mathrm{pH}$, simple tube structure, and rapid digestion that prevent gut colonization by bacterial uptake with food (Hammer et al., 2017). In agreement with this hypothesis, we observed the absence of resident microbiota. Although we found about 20 OTUs present in at least $50 \%$ of the samples, we were unable to find a core microbiome, and no OTU was shared by all the analyzed samples. The lack of a resident microbiota was also described in caterpillar from other species such as Choristoneura fumiferana (Landry et al., 2015), and also by Hammer et al. (2017) in a study analyzing the gut microbiota of several Lepidoptera species.

Several Lepidoptera studies revealed differences in microbiota community between male and female adults. Spodoptera litorallis adults differ in their microbiota community composition, since female microbiota is composed mainly of Enterococcus, Klebsiella, and Pantoea genera, while the male microbiota is dominated by Klebsiella (Chen et al., 2016). van Schooten et al. (2018) also reported significant abundance differences between sexes in several Heliconius species, but only for 13 rare OTUs, as similarly occurs for a few Melitaea cinxia larvae taxa (Minard et al., 2019). Recent S. exigua studies by Gao et al. (2019) described the absence of differences in microbiota composition between male and female adults. In our study we compare for the first time the microbiota composition between males and females at the larval stage, and the results showed no sex influence on caterpillar gut bacterial composition, diversity, or richness (Figure 4). Whether this lack of differences can be attributed to the larval stage or to the studied species would need further investigation.

Previous studies in our laboratory revealed that baculovirus infection increases the gut microbiota load in S. exigua larvae, and that increase also benefits the virus, enhancing their virulence, pathogenicity, and dispersion in laboratory conditions (Jakubowska et al., 2013). However, the relationship between viral infections in the field and microbiota composition had never been addressed. As occurs for many lepidopteran field 
populations (Williams et al., 2017), S. exigua field insects are naturally infected by different viruses (Virto et al., 2014). This is the first time that SeMNPV and/or SeIV1 presence was related with S. exigua larvae microbiota composition. Interestingly, although no differences were observed in diversity or richness, the results obtained with the multivariant analysis carried out with field samples showed a different gut bacterial composition associated with the presence or absence of viral infections (Figure 5). The bacterial load also did not show differences for infected and non-infected field larvae. However, a negative correlation between bacterial load and diversity levels was observed for both groups. So, the greater total bacterial loads in the $S$. exigua gut can be explained by the increased abundance of only a few bacterial groups, as occurs with Enterococcus in laboratory S. exigua populations (Jakubowska et al., 2013). In the case of richness, a positive correlation was observed only for the viral-infected samples, which means that the larval microbiota is composed of a greater number of bacterial species. In addition, significant abundance differences were observed for 9 OTUs, which were always more prevalent in infected S. exigua larvae than in the virus-free samples. Half of these OTUs belong to genera Acinetobacter and Pedobacter, suggesting these bacterial genera could have active functions in viral-host interaction. Staphylococcus, Sphingobium, and unclassified bacteria from families Xanthomonadaceae and Enterobacteriaceae are also differentially abundant OTUs when larvae are virally infected.

Wolbachia is an intracellular bacterium commonly found in insects, and present in about $80 \%$ of lepidopteran species (Ahmed et al., 2015). Graham et al. (2012) reported that Wolbachia increased $S$. exempta susceptibility to baculovirus, becoming a potential biological control agent. However, it was absent from our field larvae samples. Wolbachia is usually found in reproductive tissues, and was described as a parasite that manipulates reproduction in Lepidoptera (Hiroki et al., 2002; Werren et al., 2008), but it can also be found in other tissues, even in the gut (Narita et al., 2007; Whitaker et al., 2016). Since our work focused on gut microbiota, we cannot discard Wolbachia presence in other larval tissues. Nevertheless, our study identified certain bacterial groups that could influence the infection cycle, and perhaps increase susceptibility to viral infections, or even trigger covert viral activation, which could be key to developing new pest control strategies through insect microbiota manipulation.

\section{CONCLUSION}

S. exigua larvae microbiota is mainly composed of Proteobacteria, Firmicutes, Bacteroidetes, and Actinobacteria phyla. Although some differences in gut bacterial composition were observed according the different diets analyzed, the results showed that

\section{REFERENCES}

Acevedo, F. E., Peiffer, M., Tan, C.-W., Stanley, B. A., Stanley, A., Wang, J., et al. (2017). Fall armyworm-associated gut bacteria modulate plant defense gut microbiota is highly variable and strongly influenced by environmental bacteria (including those acquired through diet), supporting previous studies reflecting the lack of resident microbiota in caterpillars. In addition, microbiota study related to field larvae natural infection status revealed that the microbiota composition is significantly different when larvae present covert infections, and individual taxa could be associated specifically with the infection. Our observations suggested the possibility that microbiota composition influences the infective process of these S. exigua larvae pathogens. Thus, these results offer valuable information that will be useful for insect pest management using entomopathogenic viruses.

\section{DATA AVAILABILITY STATEMENT}

The raw Illumina sequences generated and analyzed for this study can be found in the NCBI Sequence Read Archive (SRA) under BioProject number PRJNA603888.

\section{AUTHOR CONTRIBUTIONS}

MM-S and SH designed the project. MM-S conducted the research. MM-S and MC analyzed the data. MM-S and SH wrote the manuscript.

\section{FUNDING}

This work has been supported by projects from the Spanish Ministry of Science, Innovation and Universities (No. AGL2014-57752-C2-2R and RTI2018-094350-B-C32) and the VIROPLANT project which has received funding from the European Union's Horizon 2020 Research and Innovation Program under grant agreement no. 773567.

\section{ACKNOWLEDGMENTS}

We are grateful to Magda Galeano from the Koppert Biological Systems company for sending the insects from greenhouses of Almería (Spain); Adrià Mengual-Martí for supplying the larvae from laboratory colony reared on plant-based diet.

\section{SUPPLEMENTARY MATERIAL}

The Supplementary Material for this article can be found online at: https://www.frontiersin.org/articles/10.3389/fmicb. 2020.00753/full\#supplementary-material

responses. Mol. Plant Microbe Interact. 30, 127-137. doi: 10.1094/MPMI-1116-0240-R

Ahmad, M., and Arif, I. M. (2010). Resistance of beet armyworm Spodoptera exigua (Lepidoptera: Noctuidae) to endosulfan, organophosphorus and pyrethroid 
insecticides in Pakistan. Crop Prot. 29, 1428-1433. doi: 10.1016/j.cropro.2010. 07.025

Ahmed, M. Z., Araujo-Jnr, E. V., Welch, J. J., and Kawahara, A. Y. (2015). Wolbachia in butterflies and moths: geographic structure in infection frequency. Front. Zool. 12:16. doi: 10.1186/s12983-015-0107-z

Aronesty, E. (2011). ea-utils: Command-Line Tools for Processing Biological Sequencing Data. Durham, NC. Available: https://github.com/ ExpressionAnalysis/ea-utils

Belousova, I., Ershov, N., Pavlushin, S., Ilinsky, Y., and Martemyanov, V. (2019). Molecular sexing of lepidoptera. J. Insect Physiol. 114, 53-56. doi: 10.1016/j. jinsphys.2019.02.005

Berman, T. S., Laviad-Shitrit, S., Lalzar, M., Halpern, M., and Inbar, M. (2018). Cascading effects on bacterial communities: cattle grazing causes a shift in the microbiome of a herbivorous caterpillar. ISME J. 12, 1952-1963. doi: 10.1038/ s41396-018-0102-4

Brewer, M. J., and Trumble, J. T. (1989). Field monitoring for insecticide resistance in beet armyworm (Lepidoptera: Noctuidae). J. Econ. Entomol. 82, 1520-1526. doi: $10.1093 /$ jee/82.6.1520

Broderick, N., Raffa, K., Goodman, R., and Handelsman, J. (2004). Census of the bacterial community of the gypsy moth larval midgut by using culturing and culture-independent methods. Appl. Environ. Microbiol. 70, 293-300. doi: 10.1128/AEM.70.1.293

Caccia, S., Lelio, I. D., Storia, A. L., Marinelli, A., Varricchio, P., Franzetti, E., et al. (2016). Midgut microbiota and host immunocompetence underlie Bacillus thuringiensis killing mechanism. Proc. Natl. Acad. Sci. U.S.A. 113, 9486-9491. doi: $10.1073 /$ pnas. 1521741113

Caporaso, J. G., Kuczynski, J., Stombaugh, J., Bittinger, K., Bushman, F. D., Costello, E. K., et al. (2010). QIIME allows analysis of high-throughput community sequencing data. Nat. Methods 7, 335-336. doi: 10.1038/nmeth.f.303

Chaturvedi, S., Rego, A., Lucas, L. K., and Gompert, Z. (2017). Sources of variation in the gut microbial community of Lycaeides melissa caterpillars. Sci. Rep. 7:11335. doi: 10.1038/s41598-017-11781-1

Che, W., Shi, T., Wu, Y., and Yang, Y. (2013). Insecticide resistance status of field populations of Spodoptera exigua (Lepidoptera: Noctuidae) from China. J. Econ. Entomol. 106, 1855-1862. doi: 10.1603/ec13128

Chen, B., Du, K., Sun, C., Vimalanathan, A., Liang, X., Li, Y., et al. (2018). Gut bacterial and fungal communities of the domesticated silkworm (Bombyx mori) and wild mulberry-feeding relatives. ISME J. 12, 2252-2262. doi: 10.1038/ s41396-018-0174- 1

Chen, B., Teh, B. S., Sun, C., Hu, S., Lu, X., Boland, W., et al. (2016). Biodiversity and activity of the gut microbiota across the life history of the insect herbivore Spodoptera littoralis. Sci. Rep. 6:29505. doi: 10.1038/srep29505

Dillon, R. J., and Dillon, V. M. (2004). The gut bacteria of insects: nonpathogenic interactions. Annu. Rev. Entomol. 49, 71-92. doi: 10.1146/annurev.ento.49. 061802.123416

Edgar, R. C., Haas, B. J., Clemente, J. C., Quince, C., and Knight, R. (2011). UCHIME improves sensitivity and speed of chimera detection. Bioinformatics 27, 2194-2200. doi: 10.1093/bioinformatics/btr381

Elvira, S., Gorría, N., Muñoz, D., Williams, T., and Caballero, P. (2010). A simplified low-cost diet for rearing Spodoptera exigua (Lepidoptera: Noctuidae) and its effect on S. exigua nucleopolyhedrovirus production. J. Econ. Entomol. 103, 17-24. doi: 10.1603/EC09246

Engel, P., and Moran, N. A. (2013). The gut microbiota of insects - diversity in structure and function. FEMS Microbiol. Rev. 37, 699-735. doi: 10.1111/15746976.12025

Gao, X., Li, W., Luo, J., Zhang, L., Ji, J., Zhu, X., et al. (2019). Biodiversity of the microbiota in Spodoptera exigua (Lepidoptera: Noctuidae). J. Appl. Microbiol. 126, 1199-1208. doi: 10.1111/jam.14190

Gasmi, L., Martínez-Solís, M., Frattini, A., Ye, M., Collado, M. C., Turlings, T. C. J., et al. (2019). Can herbivore-induced volatiles protect plants by increasing the herbivores' susceptibility to natural pathogens? Appl. Environ. Microbiol. 85:e01468-18.

Graham, R. I., Grzywacz, D., Mushobozi, W. L., and Wilson, K. (2012). Wolbachia in a major African crop pest increases susceptibility to viral disease rather than protects. Ecol. Lett. 15, 993-1000. doi: 10.1111/j.1461-0248.2012.01820.x

Hammer, T. J., and Bowers, M. D. (2015). Gut microbes may facilitate insect herbivory of chemically defended plants. Oecologia 179, 1-14. doi: 10.1007/ s00442-015-3327-1
Hammer, T. J., Janzen, D. H., Hallwachs, W., Jaffe, S. P., and Fierer, N. (2017). Caterpillars lack a resident gut microbiome. Proc. Natl. Acad. Sci. U.S.A. 114, 9641-9646. doi: 10.1073/pnas.1707186114

Hannula, S. E., Zhu, F., Heinen, R., and Bezemer, T. M. (2019). Foliar-feeding insects acquire microbiomes from the soil rather than the host plant. Nat. Commun. 10:1254. doi: 10.1038/s41467-019-09284-w

Hernández-Martínez, P., Naseri, B., Navarro-Cerrillo, G., Escriche, B., Ferré, J., and Herrero, S. (2010). Increase in midgut microbiota load induces an apparent immune priming and increases tolerance to Bacillus thuringiensis. Environ. Microbiol. 12, 2730-2737. doi: 10.1111/j.1462-2920.2010.02241.x

Hiroki, M., Kato, Y., Kamito, T., and Miura, K. (2002). Feminization of genetic males by a symbiotic bacterium in a butterfly, Eurema hecabe (Lepidoptera: Pieridae). Naturwissenschaften 89, 167-170. doi: 10.1007/s00114-002-0303-5

Jakubowska, A. K., Vogel, H., and Herrero, S. (2013). Increase in gut microbiota after immune suppression in baculovirus-infected larvae. PLoS Pathog. 9:e1003379. doi: 10.1371/journal.ppat.1003379

Jones, A. G., Mason, C. J., Felton, G. W., and Hoover, K. (2019). Host plant and population source drive diversity of microbial gut communities in two polyphagous insects. Sci. Rep. 9:2792. doi: 10.1038/s41598-019-39163-9

Klindworth, A., Pruesse, E., Schweer, T., Rg Peplies, J., Quast, C., Horn, M., et al. (2013). Evaluation of general 16S ribosomal RNA gene PCR primers for classical and next-generation sequencing-based diversity studies. Nucleic Acids Res. 41:e1. doi: 10.1093/nar/gks808

Koike, Y., Mita, K., Suzuki, M. G., Maeda, S., Abe, H., Osoegawa, K., et al. (2003). Genomic sequence of a $320-\mathrm{kb}$ segment of the $\mathrm{Z}$ chromosome of Bombyx mori containing a kettin ortholog. Mol. Genet. Genomics 269, 137-149. doi: 10.1007/s00438-003-0822-6

Landry, M., Comeau, A. M., Derome, N., Cusson, M., and Levesque, R. C. (2015). Composition of the spruce budworm (Choristoneura fumiferana) midgut microbiota as affected by rearing conditions. PLoS One 10:e0144077. doi: 10 . 1371/journal.pone.0144077

Livak, K. J., and Schmittgen, T. D. (2001). Analysis of relative gene expression data using real-time quantitative PCR and the 2- $\Delta \Delta$ CT method. Methods 25, 402-408. doi: 10.1006/meth.2001.1262

Mason, C. J., Rubert-Nason, K. F., Lindroth, R. L., and Raffa, K. F. (2015). Aspen defense chemicals influence midgut bacterial community composition of gypsy moth. J. Chem. Ecol. 41, 75-84. doi: 10.1007/s10886-014-0530-1

Mereghetti, V., Chouaia, B., and Montagna, M. (2017). New insights into the microbiota of moth pests. Int. J. Mol. Sci. 18:2450. doi: 10.3390/ijms 18112450

Millán-Leiva, A., Jakubowska, A. K., Ferré, J., and Herrero, S. (2012). Genome sequence of SeIV-1, a novel virus from the Iflaviridae family infective to Spodoptera exigua. J. Invertebr. Pathol. 109, 127-133. doi: 10.1016/j.jip.2011.10. 009

Minard, G., Tikhonov, G., Ovaskainen, O., and Saastamoinen, M. (2019). Variation in Melitaea cinxia gut microbiota is phylogenetically highly structured but only mildly driven by host plant microbiota, sex or parasitism. bioRxiv [Prepint] doi: 10.1101/510446

Moulton, J. K., Pepper, D. A., and Dennehy, T. J. (2000). Beet armyworm (Spodoptera exigua) resistance to spinosad. Pest Manag. Sci. 56, 842-848. doi: 10.1002/1526-4998(200010)56:10<842::aid-ps212>3.0.co;2-h

Nadkarni, M. A., Martin, F. E., Jacques, N. A., and Hunter, N. (2002). Determination of bacterial load by real-time PCR using a broad-range (universal) probe and primers set. Microbiology 148(Pt 1), 257-266. doi: 10. 1099/00221287-148-1-257

Narita, S., Nomura, M., and Kageyama, D. (2007). Naturally occurring single and double infection with Wolbachia strains in the butterfly Eurema hecabe: transmission efficiencies and population density dynamics of each Wolbachia strain. FEMS Microbiol. Ecol. 61, 235-245. doi: 10.1111/j.1574-6941.2007. 00333.x

Osorio, A., Martínez, A. M., Schneider, M. I., Díaz, O., Corrales, J. L., Avilés, M. C., et al. (2008). Monitoring of beet armyworm resistance to spinosad and methoxyfenozide in Mexico. Pest Manag. Sci. 64, 1001-1007. doi: 10.1002/ps. 1594

Phalnikar, K., Kunte, K., and Agashe, D. (2018). Dietary and developmental shifts in butterfly-associated bacterial communities. R. Soc. Open Sci. 5:171559. doi: 10.1098/rsos.171559

Raymond, B., Johnston, P. R., Wright, D. J., Ellis, R. J., Crickmore, N., and Bonsall, M. B. (2009). A mid-gut microbiota is not required for the pathogenicity of 
Bacillus thuringiensis to diamondback moth larvae. Environ. Microbiol. 11, 2556-2563. doi: 10.1111/j.1462-2920.2009.01980.x

Schmieder, R., Edwards, R., and Bateman, A. (2011). Quality control and preprocessing of metagenomic datasets. Bioinformatics 27, 863-864. doi: 10. 1093/bioinformatics/btr026

Shao, Y., Spiteller, D., Tang, X., Ping, L., Colesie, C., Münchberg, U., et al. (2011). Crystallization of $\alpha$ - and $\beta$-carotene in the foregut of Spodoptera larvae feeding on a toxic food plant. Insect Biochem. Mol. Biol. 41, 273-281. doi: 10.1016/j. ibmb.2011.01.004

Suzuki, M. G., Shimada, T., and Kobayashi, M. (1999). Bm kettin, homologue of the Drosophila kettin gene, is located on the $\mathrm{Z}$ chromosome in Bombyx mori and is not dosage compensated. Heredity 82, 170-179. doi: 10.1038/sj.hdy.6884570

van Schooten, B., Godoy-Vitorino, F., McMillan, W. O., and Papa, R. (2018). Conserved microbiota among young Heliconius butterfly species. PeerJ 6:e5502. doi: $10.7717 /$ peerj. 5502

Virto, C., Navarro, D., Tellez, M. M., Herrero, S., Williams, T., Murillo, R., et al. (2014). Natural populations of Spodoptera exigua are infected by multiple viruses that are transmitted to their offspring. J. Invertebr. Pathol. 122, 22-27. doi: 10.1016/j.jip.2014.07.007

Werren, J. H., Baldo, L., and Clark, M. E. (2008). Wolbachia: master manipulators of invertebrate biology. Nat. Rev. Microbiol. 6, 741-751. doi: 10.1038/ nrmicro1969

Whitaker, M. R. L., Salzman, S., Sanders, J., Kaltenpoth, M., and Pierce, N. E. (2016). Microbial communities of lycaenid butterflies do not correlate with larval diet. Front. Microbiol. 7:1920. doi: 10.3389/fmicb.2016.01920

Williams, T., Virto, C., Murillo, R., and Caballero, P. (2017). Covert infection of insects by baculoviruses. Front. Microbiol. 8:1337. doi: 10.3389/fmicb.2017. 01337

Xia, X., Gurr, G. M., Vasseur, L., Zheng, D., Zhong, H., Qin, B., et al. (2017). Metagenomic sequencing of diamondback moth gut microbiome unveils key holobiont adaptations for herbivory. Front. Microbiol. 8:663. doi: 10.3389/ fmicb.2017.00663

Xia, X., Zheng, D., Zhong, H., Qin, B., Gurr, G. M., Vasseur, L., et al. (2013). DNA sequencing reveals the midgut microbiota of diamondback moth, Plutella xylostella (L.) and a possible relationship with insecticide resistance. PLoS One 8:e68852. doi: 10.1371/journal.pone.00 68852

Xiang, H., Wei, G. F., Jia, S., Huang, J., Miao, X. X., Zhou, Z., et al. (2006). Microbial communities in the larval midgut of laboratory and field populations of cotton bollworm (Helicoverpa armigera). Can. J. Microbiol. 52, 1085-1092. doi: 10.1139/W06-064

Yun, J.-H., Roh, S. W., Whon, T. W., Jung, M.-J., Kim, M.-S., Park, D.-S., et al. (2014). Insect gut bacterial diversity determined by environmental habitat, diet, developmental stage, and phylogeny of host. Appl. Environ. Microbiol. 80, 5254-5264. doi: 10.1128/AEM.01226-14

Zakrzewski, M., Proietti, C., Ellis, J. J., Hasan, S., Brion, M.-J., Berger, B., et al. (2017). Calypso: a user-friendly web-server for mining and visualizing microbiome-environment interactions. Bioinformatics 33, 782-783. doi: 10 . 1093/bioinformatics/btw725

Conflict of Interest: The authors declare that the research was conducted in the absence of any commercial or financial relationships that could be construed as a potential conflict of interest.

Copyright (C) 2020 Martínez-Solís, Collado and Herrero. This is an open-access article distributed under the terms of the Creative Commons Attribution License (CC BY). The use, distribution or reproduction in other forums is permitted, provided the original author(s) and the copyright owner(s) are credited and that the original publication in this journal is cited, in accordance with accepted academic practice. No use, distribution or reproduction is permitted which does not comply with these terms. 